\title{
Gain Calculation in a Quantum Well Laser Simulator Using an Eight Band k·p Model
}

\author{
F. OYAFUSO, P. VON ALLMEN, M. GRUPEN and K. HESS
}

Beckman Institute, University of Illinois, Urbana, IL 61801

\begin{abstract}
Effects of non-parabolicity and band-warping of the energy dispersion are entered in a quantum well laser simulator (MINILASE-II), which self-consistently solves Schödinger's equation, Poisson's equation, the drift diffusion equations, and the photon rate equations. An eight band k.p model is used to determine the electronic band structure for a strained-layer $\mathrm{In}_{2} \mathrm{Ga}_{8} \mathrm{As} / \mathrm{Al}_{1} \mathrm{Ga}_{9} \mathrm{As}$ system. The $\mathbf{k} \cdot \mathbf{p}$ calculation is performed independently of the laser simulator, and exported to MINILASE-II in the form of a density of states and an energydependent averaged momentum matrix element. The results obtained for the gain and modulation response are compared to those obtained from a parabolic band model with a constant matrix element.
\end{abstract}

Keywords: gain, k · p, laser, quantum well, modulation response, bandstructure

In recent years, growing computational resources have made it possible to develop sophisticated semiconductor laser simulators, such as MINILASE-II [1], that provide a better understanding of the physical processes involved. MINILASE-II solves simultaneously the drift diffusion equations, including thermionic emission and quantum well (QW) capture, Poisson's equation and the photon rate equations for arbitrary two dimensional geometries. An accurate description of the gain still requires knowledge of the QW energy bandstructure. In this paper we explain how we connect an accurate description of the electronic bandstructure obtained from a multiband $\mathbf{k} \cdot \mathbf{p}$ theory [2] with the simulator MINILASE-II and discuss the resulting gain and modulation response of a strained-layer $\mathrm{In}_{.2} \mathrm{Ga}_{.8} \mathrm{As} / \mathrm{Al}_{.1} \mathrm{Ga}_{.9} \mathrm{As}$ quantum well laser.

The k.p Hamiltonian at the center of the Brillouin zone is written as follows,

$$
\begin{aligned}
& H_{n l}\left(\mathbf{k}_{\|}, k_{z}\right)=\left(E_{n}(0)+\frac{\hbar^{2}\left(\mathbf{k}_{\|}^{2}+k_{z}^{2}\right)}{2 m}\right) \delta_{n l} \\
& +\left(\mathbf{k}_{\|}+k_{z} \hat{\mathrm{e}}_{\mathrm{z}}\right) \cdot \mathrm{P}_{n l}+\sum_{\alpha \beta} \Gamma_{n l}^{\alpha \beta} k_{\alpha} k_{\beta}+H_{n l}^{\text {(strain })}
\end{aligned}
$$

where the indices run over the bands in our basis set $\mathrm{B}, \mathbf{P}_{n l}$ are the momentum matrix elements, $\Gamma_{n l}^{\alpha \beta}$ are renormalization constants describing the contribution from bands not contained in $\mathrm{B}$, and $H^{(\text {strain) }}$ describes the strain, assumed to be confined to the well region. The number of bands included in B determines the fraction of the Brillouin zone that is accurately described. Since lasing involves transitions between states near $\Gamma$, it is sufficient for III-V compounds with zincblende symmetry to include the heavy hole $(\mathrm{HH})$ and light hole bands $(\mathrm{LH})\left(\Gamma_{8}^{v}\right)$, the split-off bands (SO) $\left(\Gamma_{7}^{v}\right)$ and the lowest conduction bands $\left(\Gamma_{6}^{v}\right)$. The resulting $8 \times 8$ Hamiltonian has been described in the literature $[3,4]$. 
The quantum well layers are assumed to be grown in the $z$ direction and the usual substitution $k_{z} \rightarrow \partial_{z} / i$ is made to obtain the effective mass equations,

$$
\sum_{l \in B} H_{m l}\left(\mathbf{k}_{\|}, \partial_{z} / i\right) \phi_{l}^{\left(n \mathbf{k}_{\|} k_{q}\right)}(z)=E^{(n)} \phi_{m}^{\left(n \mathbf{k}_{\|} k_{q}\right)}(z),
$$

where $k_{q}$ is the wave number for the $1 \mathrm{D}$ Brillouin zone of the superlattice, and the band parameters take different values in the well and the barrier regions [2]. $\phi_{m}^{\left(n \mathbf{k} \| k_{q}\right)}(z)$ are the superlattice envelope functions which are expanded in plane waves in the $\mathrm{z}$ direction. The wave functions, needed to compute the k-dependent optical matrix elements, $\mathbf{M}_{n n}$, $\left.=<\psi_{n k \|}|\mathbf{P}| \psi_{n^{\prime} k \|}\right\rangle$, have the following form:

$$
\begin{aligned}
\psi_{n \mathbf{k}_{\|}} & =\exp \left(i \mathbf{k}_{\|} \cdot \mathbf{r}\right) \\
& \sum_{m \in \mathrm{B}}\left(u_{m 0}+\sum_{l \notin \mathrm{B}} \frac{\mathbf{k}_{\|} \cdot \mathbf{P}_{l m}}{E_{l}(0)-E_{m}(0)} u_{l 0}\right) \phi_{m}^{\left(n \mathbf{k}_{\|} k_{q}\right)}(z)
\end{aligned}
$$

In principle, Poisson's equation, the $\mathbf{k} \cdot \mathbf{p}$ equations, and the rate equations are coupled together in a completely self-consistent manner. However, it is impractical to perform a complete $\mathbf{k} \cdot \mathbf{p}$ calculation within MINILASE-II at each iteration for the electric field and the distribution functions. We therefore assume flat bands for the $\mathbf{k} \cdot \mathbf{p}$ calculation, and the resulting density of states (DOS) and optical matrix elements (OME) are exported to the laser simulator. MINILASE-II then self-consistently solves the Schrödinger equation for the QW, Poisson's equation including the unconfined carriers, and the rate equations, in the presence of the external electric field. More in detail, the DOS are pinned to the lowest CB and VB subbands edges to evaluate the $\mathrm{QW}$ contribution, $\rho_{Q W}$, to the charge density. For example, for the $\mathrm{CB}$, the electron density is given by

$$
\rho_{Q W, c}=\sum_{\varepsilon_{n}} g_{c}\left(\varepsilon_{n}-E_{c}^{1}(0)+\tilde{E}_{c}\right) \tilde{f}_{e}\left(\varepsilon_{n}\right)\left|\phi_{c}\right|^{2},
$$

where $g_{c}\left(\varepsilon_{n}\right)$ is the total DOS for all the conduction subbands, obtained from the $\mathbf{k} \cdot \mathbf{p}$ calculation, $\phi_{\mathrm{c}}$ is the first eigenstate of the Schrödinger equation solved within MINILASE-II, using an effective mass determined from the k.p calculation, and $E_{c}^{1}(0)$ and $\tilde{E}_{c}$ are the first subband edges computed with the $\mathbf{k} \cdot \mathbf{p}$ model and in MINILASE-II, respectively. The summation is made over an energy grid determined in MINILASE-II, and $\tilde{f}_{e}$ is the non-equilibrium distribution function also obtained within MINILASE-II. We have assumed in eq (4) that the subband dispersions and the separation between subband edges do not depend on the electric field in the QW, since in the lasing regime the unconfined carriers efficiently screen the applied electric field. Also, the higher subbands are assumed to be almost empty so that it is sufficient to use only the wave function of the first subband in $\rho_{Q W}$. We can easily improve the expression for $\rho_{Q W}$ by adding a summation over the number of subbands and by taking a different DOS for each subband.

An unbroadened gain coefficient is obtained from the following expression,

$$
g^{(0)}(\Omega) \approx \sum_{n}\left(\tilde{f}_{e}\left(\varepsilon_{n}\right)+\tilde{f}_{h}\left(\varepsilon_{n}-\Omega\right)-1\right) P_{n}(\Omega),
$$

where

$$
\begin{aligned}
P_{n}(\Omega) & =\sum_{i j} \int \frac{d^{2} k_{\|}}{(2 \pi)^{2}}\left|\hat{\mathrm{e}} \cdot \mathbf{M}_{i j}\left(\mathbf{k}_{\|}\right)\right|^{2} \\
& \eta_{n}\left(E_{c}^{i}\left(\mathbf{k}_{\|}\right)\right) \delta\left(E_{c}^{i}\left(\mathbf{k}_{\|}\right)-E_{\mathcal{v}}^{j}\left(\mathbf{k}_{\|}\right)-\Omega\right)
\end{aligned}
$$

is a matrix element factor calculated separately from and is an input for MINILASE-II. The direction of the light polarization is $\hat{\mathbf{e}}, \tilde{f}_{e}$ and $\tilde{f}_{h}$ are the non-equilibrium electron and hole distribution functions, respectively, and $\eta_{n}$ is a hat function with support on the energy range $\left[\varepsilon_{n-1}, \varepsilon_{n+1}\right]$. The sum extends over all conduction subbands $i$ and valence subbands $j$. Broadening is included in a phenomenological way assuming it is caused by a scattering rate of $\tau=0.1 \mathrm{ps}$.

The k·p calculation is performed for a superlattice consisting of $\mathrm{Al}_{1} \mathrm{Ga}_{9} \mathrm{As}$ barriers of $100 \AA$, and $\mathrm{In}_{.2} \mathrm{Ga}_{8} \mathrm{As}$ wells of $80 \AA$. The barriers are wide enough to decouple adjacent wells so that the superlattice is in practice a collection of independent QW. The band parameters, $P_{n l}$ and $\Gamma_{n l}^{\alpha \beta}$ are obtained from physical parameters (Table I) with the procedure described in ref. [2, 5]. We assume a fraction conduction band discontinuity of .70. Figures 1 (a)-1(b) show the unbroadened DOS for the conduction and the 
TABLE I Material Parameters used in the $\mathbf{k} \cdot \mathbf{p}$ calculation

\begin{tabular}{lcccccccc}
\hline material & $\gamma 1$ & $\gamma 2$ & $\gamma 3$ & $m_{c} / m_{o}$ & $E_{g}(\mathrm{eV})$ & $\Delta_{0}(\mathrm{eV})$ & $\Delta E_{\text {hydro }}(\mathrm{eV})$ & $\Delta E_{\text {shear }}(\mathrm{eV})$ \\
\hline $\mathrm{Al}_{.}{ }_{1} \mathrm{Ga} .{ }_{9} \mathrm{As}$ & 6.49 & 1.95 & 2.73 & 0.0713 & 1.5396 & 0.3342 & & \\
$\mathrm{In}_{2} \mathrm{Ga}_{.} \mathrm{As}$ & 9.56 & 3.34 & 4.14 & 0.0551 & 1.1351 & 0.3488 & 0.1826 & -0.0539 \\
\hline
\end{tabular}

valence subbands. We recognize the steps typical of a $2 \mathrm{D}$ confined system. The structures on the plateau are due to the non-parabolicity of the subband dispersion. Figures 1(c)-1(d) show the OME dispersion for TE polarization in the [110] direction and two directions of $\mathbf{k}_{\|}$, parallel to $\hat{\mathbf{e}}$ and at $45^{\circ}$. The strongest coupling near $\mathbf{k}_{\|}=0$ is between $\mathrm{CB} 1$ and $\mathrm{HH} 1$ as is expected for this polarization from the form of the HH Bloch function. The OME decreases as the $\mathbf{k}$ component parallel to $\hat{\mathbf{e}}$ increases (for $\mathbf{k}_{\|}=0$, $\mathbf{k}$ is characterized by a finite $\mathbf{k}_{z}$ ), and the decrease is fastest for $\mathbf{k}_{\|}$parallel to $\hat{\mathbf{e}}[6]$.
We now describe the results obtained from MINILASE-II for an operating regime beyond the lasing threshold. Figure 2(a) shows the optical gain spectra for various implementations of the DOS and OME. The maximum height of the gain is pinned by the losses in the laser which are the same for all three implementations. For the curve labeled "KP", the DOS and the OME are imported from the k'p calculation in the manner described above. For the other curves the DOS are determined by the effective masses of $\mathrm{CB} 1$ and $\mathrm{HH} 1$ at $\Gamma$, and the OME are con-
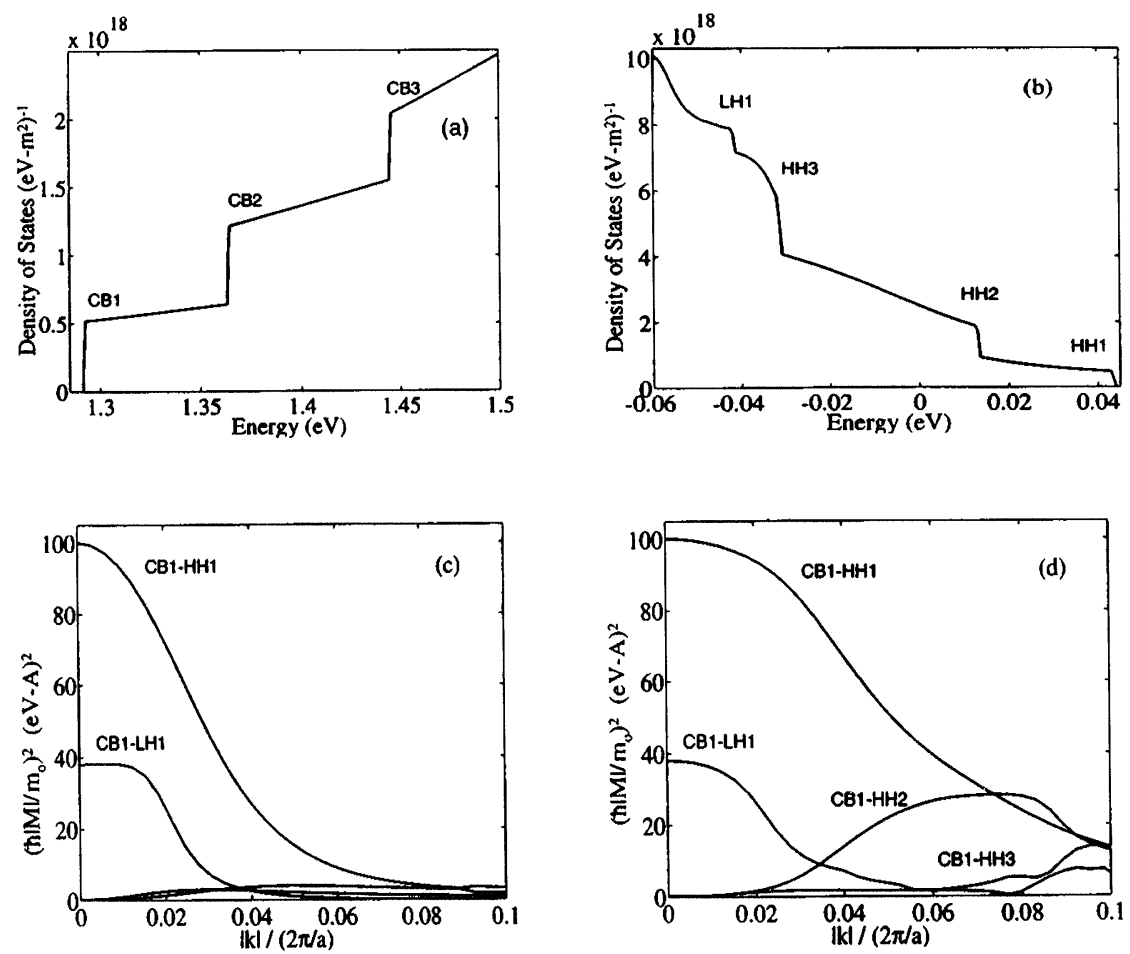

FIGURE 1 (a) and (b) DOS for the CB and VB subbands, respectively. (c) and (d) optical matrix elements for $k \|$ in the [110] and [100] directions respectively. Transitions between $\mathrm{CB} 1$ and the valence bands corresponding to bound states at $\Gamma$ are shown. The polarization is in the [110] direction 
stants derived from averaging the OME for a CB-HH transitions over the directions of $\mathbf{k}$ in a small neighborhood of $\Gamma$. For the curve labeled "2D", the OME $\left(\left|\overline{M_{2 D}}\right|^{2}\right)=P^{2}$ is averaged over $\mathbf{k}_{\|}$, where $P=<$ $S\left|p_{x}\right| X>$ is the band parameter used for the k'p calculation. The $\mathrm{QME}$ for the curve labeled "3D" $\left(\left|\overline{M_{3 D}}\right|^{2}=\frac{2}{3} P^{2}\right)$ is obtained from an average over all directions of $\mathbf{k}$. For the last curve, labeled "KANE", a $3 \mathrm{D}$ average over $\mathbf{k}$ is used, and $P$ is obtained from a 3band Kane model [7], coupling the CB, LH and SO bands. The resulting value of $P, 9.26 \mathrm{eV}$ - $\AA$, is smaller than the value used for the $\mathbf{k} \cdot \mathbf{p}$ calculation, $10.23 \mathrm{eV}$ Å. Fig. 2(a) shows that for increasing constant OME, both the lasing frequency and transparency energy are reduced, since a larger OME requires a smaller separation between the electron and hole quasi-Fermi levels to achieve a fixed gain. The $2 \mathrm{D}$ average overestimates the "KP" result because the $\mathbf{k}$-dependent expression used for the average is valid only in a small neighborhood of $\Gamma$, and a 3D average underestimates the "KP" result.

Figure 2(b) displays the laser's response to a small square pulse in the applied voltage. The three implementations with the same DOS (KANE, 3D and 2D) give a peak shifted to larger frequencies when the OME increases. We found the population inversion to be identical for the three cases. Thus, the resonance frequency is primarily proportional to the time derivative of the photon density in the cavity which is proportional to the gain. This proportionality explains the frequency shift.

As in the case of the gain curves, one might expect the peak of the "KP" curve to lie in between the "2D" and "3D" results. However, the frequency peak is shifted to a smaller frequency than expected. This shift is due to the larger DOS for the "KP" implementation relative to the other implementations, resulting in a smaller population inversion factor. The height of the resonance in the three constant OME implementations decreases with increased OME because the laser is increasingly overdamped. The height of the resonance is larger for the "KP" implementation because the capture process is more efficient in the VB due to the smaller quasi-Fermi level and the larger DOS.
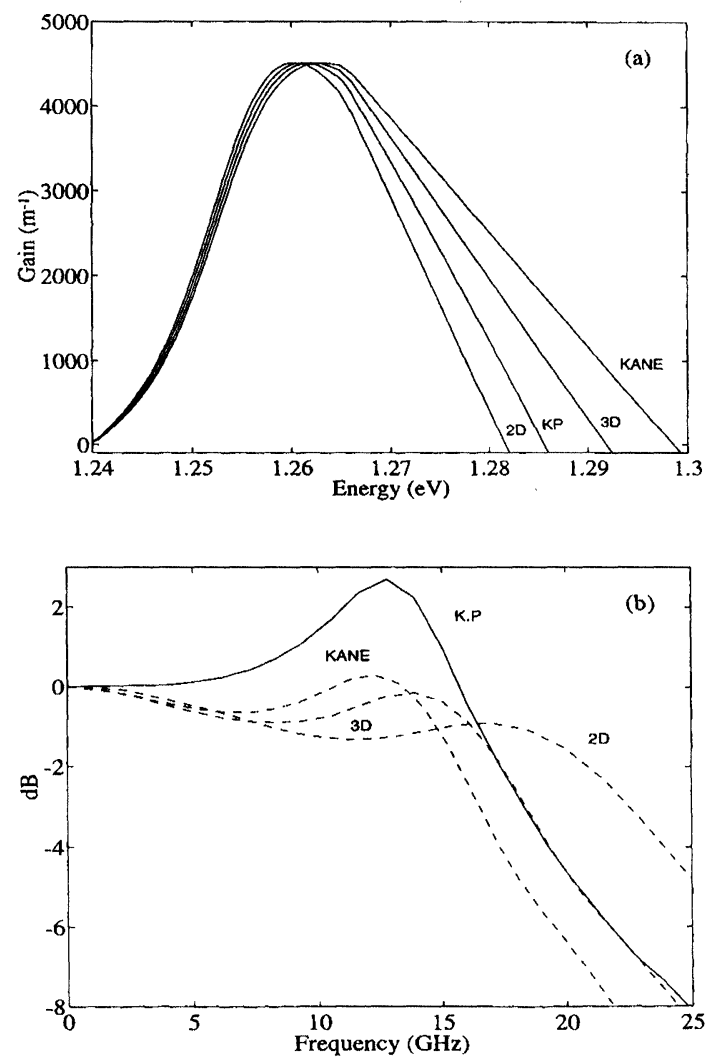

FIGURE 2 (a) Gain and (b) modulation response for four optical matrix elements. KANE, 3D, and 2D are constant matrix elements. K.P is the k-dependent matrix element determined from the eight band $\mathbf{k} \cdot \mathbf{p}$ calculation

In conclusion, we described how an accurate k·p calculation was connected to a sophisticated laser simulator and found that the transparency energy in the gain spectra and the resonance frequency in the modulation response are affected if compared to less accurate models for the DOS and the OME.

We acknowledge the support of NSF through NCCE and of the Office of Naval Research.

\section{References}

[1] M. Grupen, K. Hess, and L. Rota, "Simulating Spectral Hole Burning and the Modulation Response of Quantum Well Laser Diodes", Proc. of the SPIE Conf., 6-9 February 1995, San Jose, California.

[2] P. von Allmen, "Conduction Subbands in a GaAs/Al $\mathrm{Ga}_{1-x} \mathrm{As}$ Quantum Well: Comparing Different k·p models", Phys. Rev. $B$ 46, 15382, December 15, 1992. 
[3] M. Cardona, N. E. Christensen, and G. Fasol, "Relativistic Band Structure and Spin Orbit Splitting of Zinc-blende-type Semiconductors", Phys. Rev. B 38, 1806, July 15, 1988.

[4] E. P. O'Reilly, "Valence Band Engineering in Strained-Layer Structures", Semicond. Sci. Technol. 4, 121, 1989.

[5] C. Hermann and C. Weisbuch, "k·p Perturbation Theory in III-V Compounds and Alloys: a Reexamination", Phys. Rev. $B$ 15, 823, January $15,1977$.

[6] Corzine, S. W., Yan R., Coldren L., "Optical Gain in III-V Bulk and Quantum Well Semiconductors" in Quantum Well Lasers (edited by Zory, P.S.), Academic Press, 1993.

[7] E. O. Kane, "Band Structure of Indium Antimonide", J. Phys. Chem. Solids 1, 249, 1957.

\section{Biographies}

Fabiano Oyafuso received B.A. degrees in mathematics (with honors) and physics from UC Berkeley in 1992. He is currently pursuing the Ph.D. degree in physics at the University of Illinois at Urbana-Champaign. His thesis involves the study of the temperature dependence of theshold current densities in quantum well lasers. email: fabiano@uiuc.edu

Paul von Allmen obtained his B.S. and Ph.D. in physics from the Swiss Federal Institute of Technology in Lausanne. He joined the Zurich IBM research laboratory as a postdoctoral research associate in 1990. Since 1992 he has been an invited scholar at the Beckman Institute for Advanced Science and Technology at the University of Illinois in Urbana-Champaign. His interests range from subband structures and many-body effects in confined electron systems to the dynamical properties of nanostructures and sur- faces. His main field of interest is presently the study of the desorption mechanism of hydrogen and deuterium from a silicon surface and the related isotope effect which has important consequences for the resistance of MOS-transistors against hot carrier degradation. email: vonallme@ceg.uiuc.edu

Matt Grupen received his B.S. from Penn State University in Engineering Science in 1985. He then attended UCLA where he received an M.S. in Electron Device Physics in 1989. In 1994, he received a Ph.D. in Computational Electronics at the University of Illinois, where he currently holds a post-doctoral position. email: grupen@ceg.uiuc.edu

Karl Hess has dedicated the major portion of his research career to the understanding of electronic current flow in semiconductors and semiconductor devices with particular emphasis on effects pertinent to microchip technology. His theories and use of large computer resources are aimed at complex problems with clear applications and relevance to miniaturization of electronics. He is currently the Swanlund Professor of Electrical and Computer Engineering, Adjunct Professor for Supercomputing Applications and a Research Professor in the Beckman Institute working on topics related to Molecular and Electronic Nanostructures. He has received numerous awards, for example the IEEE David Sarnoff Field Award for electronics in 1995. email: k-hess@lux1.cso.uiuc.edu 

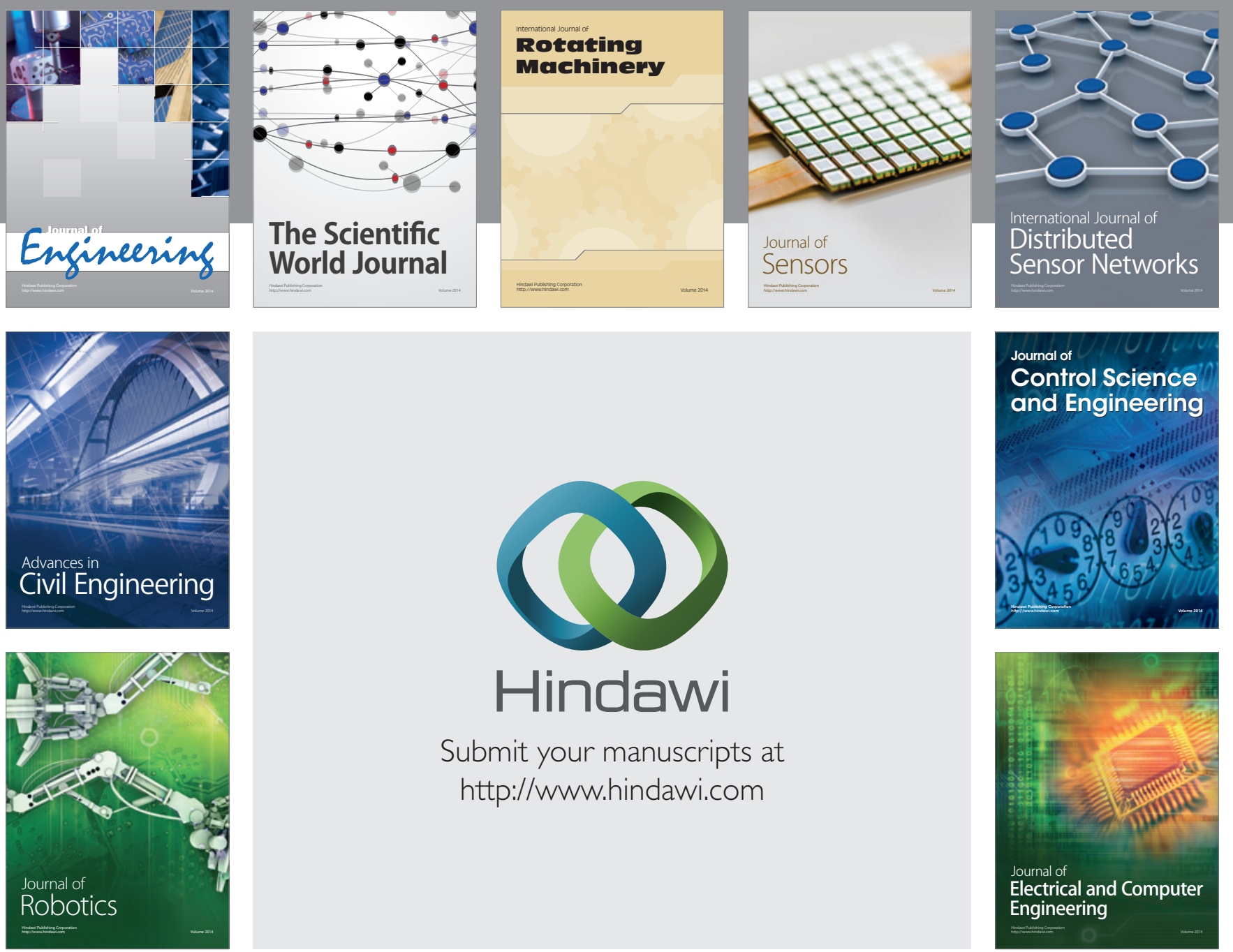

Submit your manuscripts at

http://www.hindawi.com
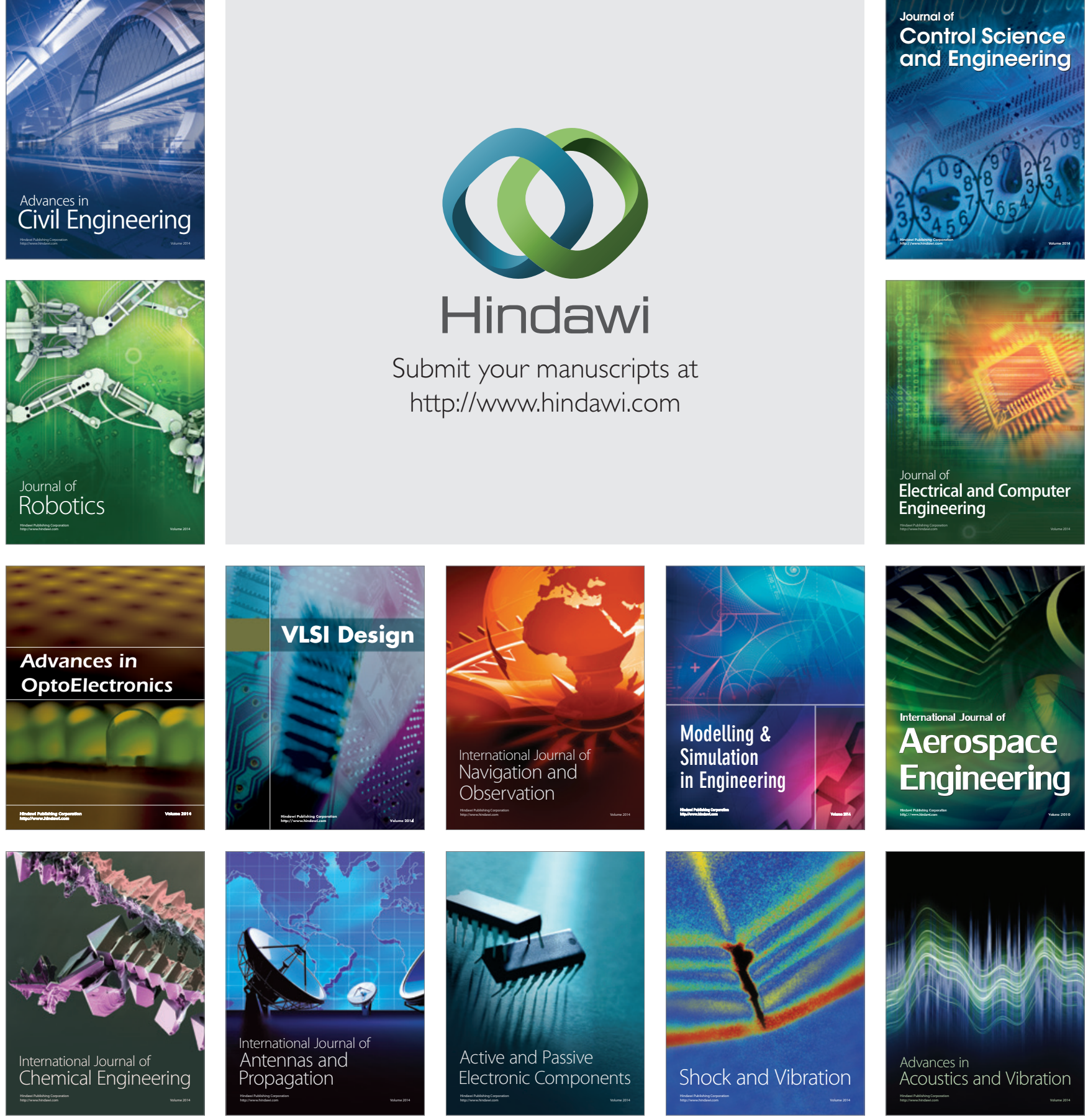